\title{
BMJ Open Development and internal validation of a simple prognostic score for early sepsis risk stratification in the emergency department
}

\author{
Bofu Liu (D) , ${ }^{1}$ Dongze Li, ${ }^{2}$ Yisong Cheng, ${ }^{2}$ Jing $Y u,{ }^{3}$ Yu Jia, ${ }^{2}$ Qin Zhang, ${ }^{2}$ \\ Yanmei Liu, ${ }^{4}$ Yu Cao ${ }^{1}$
}

To cite: Liu B, Li D, Cheng Y, et al. Development and internal validation of a simple prognostic score for early sepsis risk stratification in the emergency department. BMJ Open 2021;11:e046009. doi:10.1136/ bmjopen-2020-046009

- Prepublication history and additional supplemental material for this paper are available online. To view these files, please visit the journal online (http://dx.doi.org/10.1136/ bmjopen-2020-046009).

Received 20 0ctober 2020 Accepted 15 June 2021

Check for updates

(c) Author(s) (or their employer(s)) 2021. Re-use permitted under CC BY-NC. No commercial re-use. See rights and permissions. Published by BMJ.

${ }^{1}$ Emergency Department, Sichuan University West China Hospital, Chengdu, China ${ }^{2}$ Department of Emergency Medicine, West China Hospital, Sichuan University, Chengdu,

China

${ }^{3}$ West China School of Nursing, Sichuan University West China Hospital, Chengdu, China

${ }^{4}$ Chinese Evidence-based Medicine Center and CREAT Group, West China Hospital, Sichuan University, Chengdu, China

Correspondence to

DrYu Cao; caoyu@wchscu.cn

\section{ABSTRACT}

Objectives No validated, simple, powerful and

continuously monitorable risk prediction tools are available for patients with sepsis during the early phases in the emergency department (ED). We sought to derive a novel Simple Sepsis Early Prognostic Score (SSEPS) composed of physiological indicators that do not depend on laboratory tests and that can be used by emergency clinicians in predicting outcomes in patients with sepsis. Design Retrospective cohort analysis of a collected data source.

Participants Patients with sepsis admitted to the ED of the West China Hospital of Sichuan University between July 2015 and June 2016 were included. We excluded patients who were pregnant, those with cardiac or respiratory arrest, and those using vasoactive drugs before admission to the ED.

Primary outcome measures 28-day all-cause mortality. Results The SSEPS consisted of age, heart rate, respiratory rate and altered consciousness. Patients in the development cohort with higher SSEPS had a significantly higher mortality (first tertile vs second tertile vs third tertile: $12.5 \%$ vs $28.6 \%$ vs $53.5 \%, p<0.001$ ). The area under the receiver operating characteristic curve for SSEPS was 0.762 (95\% Cl 0.686 to 0.838$)$, which was similar to Sequential Organ Failure Assessment (SOFA) (area under the curve: $0.745,95 \% \mathrm{Cl} 0.692$ to 0.798 ) and Acute Physiology and Chronic Health Evaluation (APACHE II) (area under the curve: $0.750,95 \% \mathrm{Cl} 0.681$ to 0.819 ). Moreover, the decision curve analysis showed that the net benefit of SSEPS was higher than SOFA and APACHE II at any probability threshold.

Conclusion The SSEPS is simple and useful for clinicians in stratifying high-risk patients with sepsis at the early phase of ED admission.

\section{INTRODUCTION}

Sepsis is a fatal organ dysfunction disorder with high mortality that is caused by a dysregulated host response to infections. ${ }^{12}$ Imbalanced immune response in sepsis underlies serious organ failures and clinical outcomes. ${ }^{3}$ Early diagnoses and appropriate management of sepsis decrease the risk of adverse
Strengths and limitations of this study

- One of the strengths of the study was finding a new model developed on the basis of vital signs so as to accurately predict 28-day all-cause mortality of patients with sepsis.

- This study focuses on recognition/identification of patients at risk immediately after arrival to the emergency department.

- The new model can generally be applied to identify high-risk patients with sepsis during the early stages in the emergency department.

- Limitations of this study are its small sample size (only 236 patients in the validation group) and the lack of an external validation data set, which may limit the rigour of the study and confidence in findings.

- The variables included in the Simple Sepsis Early Prognostic Score were those recorded on admission to our emergency department.

outcomes; however, the mortality of patients with sepsis remains high. The incidence of sepsis in hospitals and intensive care units (ICUs) is $2 \%$ and $6 \%-30 \%$, respectively, and these percentages are higher in developing countries. ${ }^{4}$ Mortality from sepsis varies substantially across hospitals $(30 \%-50 \%),{ }^{56}$ suggesting the need for more accurate assessment to guide individualised treatment decision algorithms. In addition, the '2016 surviving sepsis campaign' indicated that early risk stratification and appropriate management during the initial hours after onset of symptoms improve outcomes. ${ }^{7}$ Therefore, risk stratification tools to identify high-risk patients with sepsis during the early phase of admission are critical to treatment decisions.

Although current predictive scores such as the quick Sequential Organ Failure Assessment (qSOFA), the Modified Early Warning Scoring (MEWS), the Mortality in the Emergency Department Sepsis (MEDS), the Acute 
Physiology and Chronic Health Evaluation (APACHE II), and the Sequential Organ Failure Assessment (SOFA) have been proven to have excellent risk stratification efficiency, ${ }^{2-11}$ most of them rely on data from laboratory tests or proved to be of poor validity and discriminating ability. Thus, these complex scoring systems may miss out many early critical emergency department (ED) or prehospital patients. Moreover, the conditions of patients with sepsis can change rapidly. With this in mind, traditional scoring systems cannot immediately and repeatedly assess patients during ED admission or prehospital and realise dynamic evaluation of their risk level. Therefore, a simple prognostic scoring system that does not rely on detailed medical history or invasive medical testing is needed to carry out early assessments in the ED and even before admission.

The Sepsis 3.0 consensus emphasises the organ dysfunction caused by imbalanced host responses. Studies have shown that state of consciousness and vital signs are associated with imbalance in host responses in patients with sepsis, and these variables have commonly guided the treatment of patients with sepsis. ${ }^{12}$ Thus, we hypothesised that simple physiological indicators which reflect host response during the early stages of sepsis can provide important information for evaluating the prognosis of patients. We aimed at constructing and validating a novel and instrumental independent Simple Sepsis Early Prognostic Score (SSEPS) to predict clinical adverse outcomes in patients with sepsis during ED admission or even prehospital.

\section{METHODS}

\section{Study design}

This was a single-centre, retrospective cohort study designed to derive a novel SSEPS and validate it for predicting clinical adverse outcomes in patients with sepsis during the early stages in the ED. We obtained informed consent from all participants involved. Patients and/ or the public were not involved in the design, conduct, reporting or dissemination plans of this research.

\section{Study population}

We obtained data from the West China Hospital sepsis database. Briefly, the database contains data from all adult patients ( $\geq 18$ years) diagnosed with sepsis in the ED of the West China Hospital of Sichuan University between July 2015 and June 2016. All sepsis diagnoses were based on the Sepsis-3 criteria. We excluded patients who were pregnant, those with prehospital cardiac or respiratory arrest, and those using vasoactive drugs (eg, norepinephrine, dopamine or dobutamine) (online supplemental figure 1).

\section{Development and validation sets}

To both analyse multivariate risk predictors of short-term outcomes in sepsis and test the validity of the risk predictors in a separate population, we constructed development and validation sets. Based on the development cohort data, the mortality rate is approximately $20 \%$. To satisfy this difference with $80 \%$ power at $5 \%$ significance (twotailed), assuming that the predicted value of the scoring system for death is higher than the area under the curve (AUC) value of 0.75 , at least 24 deaths are required, and the number of patients in the validation group was 120 . Therefore, we randomised all patient data into one of the two sets, with approximately $70 \%$ in the development set and $30 \%$ in the validation set. We did not use the validation set until after obtaining the results of the multivariate regression model and the predicting score.

\section{Data collection}

We collected demographic characteristics, admission vital signs, medical history, laboratory data, imaging data and final in-hospital diagnoses from the database. Laboratory data included routine haemograms with white cell count, platelet count, haemoglobin and haematocrit. We extracted serum biochemical indicators such as creatinine, urea nitrogen, cystatin-C, total bilirubin, alanine aminotransferase, aspartate aminotransferase and albumin levels. D-dimers were measured using a Sysmex CA-7000 analyser (Siemens Healthcare Diagnostics) and procalcitonin level was tested using Cobas S6000 Hitachi (Roche Diagnostics). We calculated the estimated glomerular filtration rate (eGFR) in millilitres per minute per $1.73 \mathrm{~m}^{2}$, according to the Modification of Diet in Renal Disease equation. ${ }^{13}$ The treating physicians estimated the scoring systems for sepsis, including Glasgow Coma Scale (GCS), qSOFA, MEWS, MEDS, APACHE II and SOFA, according to patients' baseline data after admission to the ED. Patients with GCS score less than 13 were identified to have altered consciousness. ${ }^{14} 15$

\section{Endpoints and follow-up}

All patients in this study were followed up for 28 days by an emergency doctor in our department using a structured telephone interview. We contacted the relatives if the patients could not be contacted directly. We obtained all other data from the hospital medical records. Our primary endpoint was 28-day all-cause mortality, and the secondary endpoint was incidence of ICU admission, mechanical ventilation and shock. ICU admission was defined as patients admitted to the ICU due to severe dystrophy, severe hydroelectrolyte balance and unstable vital signs and need for organ support. Mechanical ventilation was defined as patients who require non-invasive ventilation or invasive ventilation due to hypoxic respiratory failure. Shock was defined as patients with hypotension that persists despite adequate fluid resuscitation and requires vasopressors to support perfusion.

\section{Statistical analysis}

We used the median to replace the laboratory variables missing less than $5 \%$ and if missing variables greater than $5 \%$ were not analysed at baseline. We presented continuous variables as mean (SD) for normally 
distributed data or as median and IQR for non-normally distributed data. We carried out continuous variable analyses using an independent sample t-test or MannWhitney $\mathrm{U}$ test. We presented categorical variables as frequencies (proportions) using $\chi^{2}$ or Fisher's exact test as appropriate.

In the development cohort, we selected physiological indicators in the univariate logistic regression analyses to obtain candidate predictors of 28-day mortality and selected those for our multivariate logistic regression model. We chose age, heart rate, respiratory rate, altered consciousness, systolic blood pressure (SBP), diastolic blood pressure (DBP), oxygen saturation $\left(\mathrm{SPO}_{2}\right)$ and temperature with $\mathrm{p}<0.05$ to set up the SSEPS scoring system. There were no missing data on vital signs and consciousness, which are basic information for each admitted patients, and thus no imputation would be required for development/validation of SSEPS. We categorised SSEPS predictors based on median (IQR) cut-off values. We divided all unstandardised coefficients in the logistic regression model by the smallest coefficient to normalise them, and we set the lower risk of each variable category as the reference value group. We finally calculated the SSEPS score by a simple arithmetic sum of the integers assigned to the satisfied criteria. To examine overfitting of the model, we used the Hosmer-Lemeshow goodness of fit test and the observed and expected frequencies. We also constructed receiver operating characteristic curves and calculated the AUC for SSEPS to assess its discriminating ability as well as those of other scoring systems. We used the DeLong test to compare the AUCs of these scores, comparing the discriminating ability of SSEPS and other scores on 28-day mortality. ${ }^{16}$ We used data from the validation cohort to obtain a temporal score validation.

We analysed discriminating ability using the net reclassification improvement (NRI) and integrated discrimination improvement (IDI) to estimate the degree by which the SSEPS improved predictive ability when compared with those of other prognostic scoring systems. ${ }^{17}$ We take $5 \%$ as a cut-off value for low risk of mortality. With mortality around $20 \%$ in our study, in the NRI analysis, patients with risk of mortality less than $5 \%, 5 \%-20 \%$ and more than $20 \%$ were defined as low-risk, moderate-risk and high-risk groups, respectively. A decision curve analysis (DCA) suggested by Vickers and Elkin ${ }^{18}$ was applied to quantify the clinical usefulness of the SSEPS and to compare it with other scoring systems. We applied a Kaplan-Meier test to describe the survival estimates for our three defined risk groups (low, medium and high risk) by splitting the scoring system into tertiles. We considered two-sided $\mathrm{p}$ values $<0.05$ as statistically significant. We performed data analyses using SPSS V.21.0, Stata V.13.0 and R V.3.5.1 (R Foundation for Statistical Computing, Vienna, Austria) software.
RESULTS

\section{Patient population}

In total, we collected and analysed data from 821 patients with a mean age of $57.5 \pm 17.8$ years; $64.3 \%$ were men and 173 patients $(21.1 \%)$ died within the 28-day follow-up (online supplemental figure 1). We randomly assigned $70 \%$ of the patients to the development group $(n=585)$ and $30 \%$ to the validation group $(n=236)$. We found no significant differences in baseline characteristics, vital signs, laboratory examination values, incidence of infection site or endpoint event occurrences among the patients in both groups (online supplemental table 1).

\section{Baseline characteristics in the development group}

The development group included 585 patients $(62.4 \%$ men) with a mean age of $59.3 \pm 17.3$ years and a large proportion $(57.6 \%)$ had suspected pneumonia. Of these patients, $125(21.4 \%)$ died during the 28-day follow-up period.

Patients who died were more likely to be older, have higher respiratory rates, have higher serum lactate, urea nitrogen, creatinine, cystatin-C and eGFR levels, and have higher APACHE II, MEWS, MEDS, SOFA and qSOFA scores, in addition to lower levels of $\mathrm{pH}$, platelet count, haemoglobin, haematocrit, albumin, GCS and fibrinogen, compared with patients who survived (table 1).

\section{Logistic regression analysis for 28-day mortality in the development group}

Age, respiratory rate, heart rate, altered consciousness, $\mathrm{SBP}, \mathrm{DBP}$ and $\mathrm{SPO}_{2}$ were selected in the univariate analysis. The multivariate logistic regression analysis showed that age (OR, 1.032; 95\% CI 1.008 to $1.056 ; \mathrm{p}=0.010)$, heart rate (OR, $1.055 ; 95 \%$ CI 1.010 to $1.100 ; \mathrm{p}=0.040)$, respiratory rate $(\mathrm{OR}, 1.042 ; 95 \% \mathrm{CI} 1.003$ to 1.083 ; $\mathrm{p}=0.034)$ and altered consciousness (OR, 3.606; 95\% CI 2.296 to 5.664; $\mathrm{p}<0.001$ ) were independent predictors of 28-day sepsis mortality (table 2 ).

\section{Construction and scoring of SSEPS in the development group}

In order to construct the SSEPS, we selected independent risk factors such as age, heart rate, respiratory rate and altered consciousness in the logistic regression. These predictors were classified into quartiles. Online supplemental figure 2 shows that patients aged $\leq 46$ years and with heart rate $\leq 95$ beats per minute, respiratory frequency $\leq 21$ beats per minute or altered consciousness had a significantly lower mortality than other groups. Thus, we took the lowest risk group as reference. Table 3 shows the risk factors in the SSEPS model and the points derived for each category according to $\beta$ coefficients. The SSEPS score ranged from 0 to 21 points for 28-day sepsis mortality. We classified risks based on total scores $(0-6$ points, low; 7-9 points, medium; and 10-21 points, high); the 28-day sepsis mortality for these groups was $3.8 \%$, $13.9 \%$ and $43.6 \%$, respectively, with a statistically significant difference $(\mathrm{p}<0.001)$. 
Table 1 Comparison between survivors and non-survivors in the development cohort

\begin{tabular}{|c|c|c|c|}
\hline Variable & Dead $(n=125)$ & Alive $(n=460)$ & $P$ value \\
\hline Age, years & $63.67 \pm 15.62$ & $58.21 \pm 17.51$ & 0.050 \\
\hline Male, n (\%) & $82(65.6)$ & $283(61.5)$ & 0.404 \\
\hline Temperature, ${ }^{\circ} \mathrm{C}$ & $37.11 \pm 2.16$ & $37.33 \pm 1.05$ & 0.120 \\
\hline Heart rate, beats per minute & $111.88 \pm 20.20$ & $108.29 \pm 20.40$ & 0.081 \\
\hline Respiratory rate, beats per minute & $24.92 \pm 5.52$ & $23.26 \pm 5.19$ & 0.003 \\
\hline SBP, mm Hg & $120.18 \pm 27.22$ & $120.80 \pm 24.47$ & 0.808 \\
\hline $\mathrm{DBP}, \mathrm{mm} \mathrm{Hg}$ & $71.96 \pm 19.65$ & $73.74 \pm 16.56$ & 0.356 \\
\hline $\mathrm{SPO}_{2}, \%$ & $92.77 \pm 8.19$ & $93.98 \pm 6.86$ & 0.094 \\
\hline $\mathrm{pH}$ & $7.40 \pm 0.10$ & $7.44 \pm 0.07$ & $<0.001$ \\
\hline $\mathrm{PO}_{2}, \mathrm{~mm} \mathrm{Hg}$ & $80.83 \pm 39.23$ & $83.57 \pm 31.11$ & 0.410 \\
\hline $\mathrm{PCO}_{2}, \mathrm{~mm} \mathrm{Hg}$ & $32.68 \pm 11.56$ & $34.19 \pm 10.13$ & 0.153 \\
\hline Lactate, $\mathrm{mmol} / \mathrm{L}$ & $2.80 \pm 2.39$ & $1.76 \pm 1.27$ & $<0.001$ \\
\hline WCC, $\times 10^{9} / \mathrm{L}$ & $12.52 \pm 9.41$ & $13.17 \pm 8.05$ & 0.443 \\
\hline Platelet, $\times 10^{9} / \mathrm{L}$ & $130(64-212)$ & $166(101-251)$ & 0.001 \\
\hline Haemoglobin, g/L & $107.48 \pm 29.61$ & $113.98 \pm 29.47$ & 0.029 \\
\hline Haematocrit & $0.32 \pm 0.09$ & $0.34 \pm 0.08$ & 0.017 \\
\hline Albumin, $g / L$ & $29.09 \pm 6.29$ & $33.41 \pm 6.68$ & $<0.001$ \\
\hline Urea nitrogen, mmol/L & $8.4(5.6-14.8)$ & $6.0(4.2-9.3)$ & 0.002 \\
\hline Creatinine, $\mu \mathrm{mol} / \mathrm{L}$ & $96(62-175)$ & $75(59-106)$ & 0.041 \\
\hline eGFR, mL/min/1.73 m² & $130(65-283)$ & $95(60-159)$ & 0.049 \\
\hline Cystatin-C, mg/L & $1.78 \pm 1.17$ & $1.42 \pm 1.19$ & 0.003 \\
\hline Fibrinogen, g/L & $3.95 \pm 1.98$ & $4.54 \pm 1.61$ & 0.001 \\
\hline Total bilirubin, $\mu \mathrm{mol} / \mathrm{L}$ & $12.7(8.5-23.2)$ & $12.2(8.1-20.5)$ & 0.214 \\
\hline ALT, IU/L & $24(16-52)$ & $27(16-53)$ & 0.397 \\
\hline AST, IU/L & $42(25-65)$ & $32(21-62)$ & 0.218 \\
\hline Infection sites, n (\%) & & & 0.185 \\
\hline Lung & $81(64.8)$ & $256(55.7)$ & \\
\hline Intra-abdominal site & $24(19.2)$ & $110(23.9)$ & \\
\hline Others & $20(16.0)$ & $94(20.4)$ & \\
\hline \multicolumn{4}{|l|}{ Sepsis prognostic scores } \\
\hline GCS & $12.46 \pm 3.67$ & $14.17 \pm 2.27$ & $<0.001$ \\
\hline APACHE II & $20.30 \pm 7.37$ & $15.20 \pm 7.51$ & $<0.001$ \\
\hline MEWS & $4.70 \pm 2.24$ & $3.82 \pm 2.06$ & $<0.001$ \\
\hline MEDS & $8.92 \pm 3.76$ & $6.98 \pm 3.34$ & $<0.001$ \\
\hline SOFA & $8(6-12)$ & $4(2-6)$ & $<0.001$ \\
\hline qSOFA & $1.37 \pm 0.809$ & $0.89 \pm 0.753$ & $<0.001$ \\
\hline
\end{tabular}

ALT, aspartate aminotransferase; APACHE II, Acute Physiology and Chronic Health Evaluation; AST, alanine aminotransferase; DBP, diastolic blood pressure; eGFR, estimated glomerular filtration rate; GCS, Glasgow Coma Scale; MEDS, Mortality in the Emergency Department Sepsis; MEWS, Modified Early Warning Scoring; PCO2, Partial Pressure of Carbon Dioxide; PO2, partial pressure of oxygen; qSOFA, quick Sequential Organ Failure Assessment; SBP, systolic blood pressure; SOFA, Sequential Organ Failure Assessment; $\mathrm{SPO}_{2}$, oxygen saturation; WCC, white cell count.

\section{Goodness of fit of the SSEPS model}

Mortality ( $53.5 \%$ vs $28.6 \%$ vs $12.5 \%$ ) and incidence of ICU admission ( $76.7 \%$ vs $52.6 \%$ vs $29.5 \%$, p $<0.001)$, mechanical ventilation $(86.0 \%$ vs $53.1 \%$ vs $30.4 \%, \mathrm{p}<0.001)$ and shock $(37.2 \%$ vs $16.4 \%$ vs $5.5 \%, \mathrm{p}<0.001)$ in the high-risk group were higher than those in the moderaterisk and low-risk groups (online supplemental table 2). The Hosmer-Lemeshow goodness of fit statistic for SSEPS in the development group was 0.669 (figure 1A). The AUC of the SSEPS in predicting 28-day sepsis mortality was 
Table 2 Univariate and multivariate logistic regression analyses in the development group

\begin{tabular}{|c|c|c|c|c|c|c|}
\hline \multirow[b]{2}{*}{ Variables } & \multicolumn{3}{|c|}{ Univariate analysis } & \multicolumn{2}{|c|}{ Multivariate analysis } & \multirow{2}{*}{$\begin{array}{l}P \\
\text { value }\end{array}$} \\
\hline & OR & $95 \% \mathrm{Cl}$ & $P$ value & OR & $95 \% \mathrm{Cl}$ & \\
\hline Heart rate & 1.009 & 0.999 to 1.018 & 0.081 & 1.055 & 1.010 to 1.100 & 0.040 \\
\hline Respiratory rate & 1.052 & 1.017 to 1.088 & 0.003 & 1.042 & 1.003 to 1.083 & 0.034 \\
\hline $\mathrm{DBP}, \mathrm{mm} \mathrm{Hg}$ & 0.994 & 0.982 to 1.006 & 0.307 & 0.987 & 0.969 to 1.006 & 0.175 \\
\hline $\mathrm{SPO}_{2}, \%$ & 0.979 & 0.955 to 1.004 & 0.099 & 0.991 & 0.963 to 1.020 & 0.530 \\
\hline Temperature, ${ }^{\circ} \mathrm{C}$ & 0.902 & 0.783 to 1.039 & 0.151 & 0.898 & 0.771 to 1.047 & 0.169 \\
\hline
\end{tabular}

DBP, diastolic blood pressure; SBP, systolic blood pressure; $\mathrm{SPO}_{2}$, oxygen saturation.

0.762 (95\% CI 0.702 to $0815 ; \mathrm{p}<0.001)$, similar to those of SOFA (AUC: $0.762 ; 95 \%$ CI 0.701 to $0.814 ; \mathrm{p}>0.05$ ) and APACHE II (AUC: $0.761 ; 95 \%$ CI 0.602 to $0.814 ; \mathrm{p}>0.05$ ), higher than those of MEDS (AUC: $0.724 ; 95 \%$ CI 0.662 to $0.780 ; \mathrm{p}>0.05$ ) and qSOFA (AUC: $0.695 ; 95 \%$ CI 0.632 to $0.753 ; \mathrm{p}>0.05$ ), and higher than that of MEWS (AUC: $0.656 ; 95 \%$ CI 0.592 to $0.717 ; \mathrm{p}=0.03$; online supplemental figure 3$)$.

\section{Validation of the SSEPS model in the validation group}

In the validation group, mortality $(43.6 \%$ vs $13.9 \%$ vs $3.8 \%$ ) and incidence of ICU admission (60.3\% vs $32.9 \%$ vs $22.8 \%, \mathrm{p}<0.001)$, mechanical ventilation $(67.9 \%$ vs $29.1 \%$ vs $25.3 \%, \mathrm{p}<0.001)$ and shock $(32.1 \%$ vs $11.4 \%$ vs $6.3 \%, \mathrm{p}<0.001)$ in the high-risk group were higher than those in the moderate-risk and low-risk groups (online supplemental table 3). The Hosmer-Lemeshow goodness of fit statistic for SSEPS in the validation group was 0.522 (figure 1B). The AUC of the SSEPS in predicting 28-day sepsis mortality was 0.762 (95\% CI 0.686 to 0.838 ; $\mathrm{p}<0.001$ ), similar to those of SOFA (AUC: $0.745 ; 95 \%$ CI 0.692 to $0.798 ; \mathrm{p}>0.05$ ) and APACHE II (AUC: 0.750; $95 \%$ CI 0.681 to $0.819 ; \mathrm{p}>0.05)$ and higher than those of qSOFA (AUC: 0.695 ; 95\% CI 0.607 to 0.782 ; $\mathrm{p}<0.01$ ), MEWS (AUC: $0.656 ; 95 \%$ CI 0.567 to $0.746 ; \mathrm{p}<0.01$ ) and MEDS (AUC: $0.724 ; 95 \%$ CI 0.646 to $0.802 ; \mathrm{p}<0.01$ ) (figure 2).

Since the AUCs of the SSEPS and SOFA systems for predicting 28-day mortality were identical and the highest of all the scoring systems, we further analysed NRI, IDI and DCA to compare their discriminating abilities. SSEPS

Table 3 Logistic regression analysis of the correlations between 28-day mortality and clinical elements of patients with sepsis in the development cohort

\begin{tabular}{|c|c|c|c|c|}
\hline Variables & OR $(95 \% \mathrm{Cl})$ & $P$ value & $\beta$ coefficients & Score assigned \\
\hline$\leq 46$ & 1 & & & 0 \\
\hline $60-71$ & $1.983(1.185$ to 3.321$)$ & 0.009 & 0.685 & 4 \\
\hline$>71$ & 2.235 (1.316 to 3.796$)$ & 0.003 & 0.804 & 5 \\
\hline$\leq 95$ & 1 & & & 0 \\
\hline $95-107$ & $1.210(0.705$ to 2.077$)$ & 0.490 & 0.191 & 1 \\
\hline $107-123$ & 1.554 (0.921 to 2.622) & 0.099 & 0.441 & 3 \\
\hline$>123$ & 1.641 (0.952 to 2.829$)$ & 0.075 & 0.495 & 3 \\
\hline $22-25$ & $1.400(0.863$ to 2.271$)$ & 0.172 & 0.337 & 2 \\
\hline$>25$ & $2.787(1.742$ to 4.458$)$ & $<0.001$ & 1.025 & 6 \\
\hline Altered consciousness & 3.242 (2.182 to 4.816$)$ & $<0.001$ & 1.176 & 7 \\
\hline
\end{tabular}



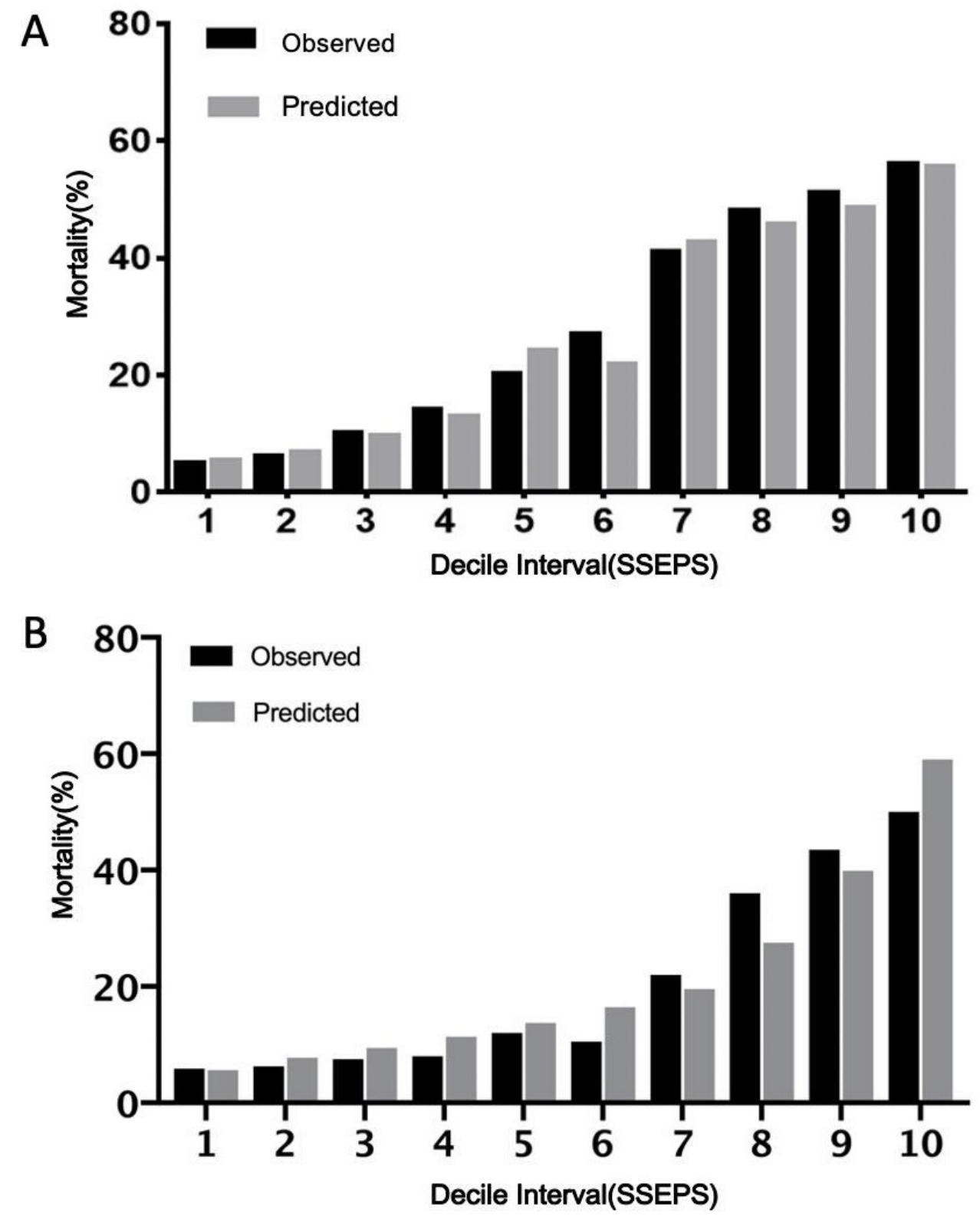

Figure 1 Calibration histogram of SSEPS for predicting 28-day sepsis mortality based on logistic regression in the development (A) and validation (B) groups. SSEPS, Simple Sepsis Early Prognostic Score.

did not show improvement in prognostic performance compared with SOFA (table 4). Six patients who died were reclassified to moderate-risk or high-risk group and seven who survived were reclassified to low-risk or moderate-risk group; thus, the NRI between SSEPS and SOFA was $-0.088(\mathrm{p}=0.42)$ and the IDI was -0.039 $(\mathrm{p}=0.27)$. However, the DCA showed that the net benefit of SSEPS was higher than those of SOFA or APACHE II at any threshold (figure 3).

\section{DISCUSSION}

In this study, we tested a simple early prognostic score for sepsis consisting of age, heart rate, respiratory rate and altered consciousness measured in the ED. Our data show that patients with higher SSEPS scores had higher risk of 28-day all-cause mortality and incidence of ICU admission, mechanical ventilation and shock. Irrespective of the severity of sepsis, the prognostic value of the SSEPS was superior to that of qSOFA, MEWS, MEDS, SOFA and APACHE II. Therefore, our SSEPS seems to be a powerful tool to stratify high-risk patients with sepsis during the early phases in the ED, and we could assess the severity of sepsis by monitoring age, heart rate, respiratory rate and altered consciousness during the early phases.

Sepsis is considered a severe and life-threatening condition due to organ dysfunctions caused by inappropriate host responses to infections. ${ }^{1}$ Each hour of delay in treatment initiation increases the risk of sepsis-related mortality by approximately $8 \% \cdot{ }^{19}$ Early and quick risk stratification plays a vital role in the early management of sepsis, and 


\begin{tabular}{|c|c|c|c|c|}
\hline \multicolumn{2}{|l|}{ Variables } & $\mathrm{ROC}(95 \% \mathrm{Cl})$ & P-value & P for comparison \\
\hline SSEPS & $\mapsto \bullet-1$ & $0.762(0.686-0.838)$ & $<0.01$ & Ref \\
\hline APACHE II & $\vdash *-1$ & $0.750(0.681-0.819)$ & $<0.01$ & $>0.05$ \\
\hline SOFA & $\mapsto \bullet-1$ & $0.745(0.692-0.798)$ & $<0.01$ & $>0.05$ \\
\hline qSOFA & $\longmapsto \bullet$ & $0.695(0.607-0.782)$ & $<0.01$ & $<0.01$ \\
\hline MEWS & $\longmapsto \longrightarrow$ & $0.656(0.567-0.746)$ & $<0.01$ & $<0.01$ \\
\hline MEDS & $\mapsto \bullet$ & $0.724(0.646-0.802)$ & $<0.01$ & $<0.01$ \\
\hline 0.2 & $\begin{array}{ll}1 & 1 \\
0.6 & 0.8\end{array}$ & & & \\
\hline
\end{tabular}

Figure 2 AUC of SSEPS and other predicting scoring systems for 28-day sepsis mortality in the validation group. APACHE II, Acute Physiology and Chronic Health Evaluation; AUC, area under the curve; MEDS, Mortality in the Emergency Department Sepsis; MEWS, Modified Early Warning Scoring; qSOFA, quick Sequential Organ Failure Assessment; Ref, reference; SOFA, Sequential Organ Failure Assessment; SSEPS, Simple Sepsis Early Prognostic Score;ROC, Receiver operating characteristic.

many prognostic scoring systems have been put in place to stratify patients with sepsis in the ED according to their risk. The qSOFA has been recommended in the guidelines for screening patients with infections for organ dysfunction, but has shown poor sensitivity and specificity in predicting 28-day mortality in ED patients with sepsis. ${ }^{20}$ Studies have also shown that MEDS and MEWS scores predict adverse outcomes in patients with sepsis, but with limited risk stratification power. ${ }^{112122}$ SOFA and APACHE II scores consist of multiple physiological and haematological variables and are powerful in predicting risk among patients with sepsis; however, they are so complex that they cannot be used to continuously monitor severity of patients outside the ICU or during the early phases in the ED. Therefore, a novel and powerful prognostic score that can be applied in the ED is needed. In our study, the novel SSEPS, which considers age, heart rate, respiratory rate and altered consciousness, could independently and continuously predict mortality and incidence of ICU

Table 4 NRI of SOFA and SSEPS in predicting 28-day sepsis mortality in the validation group

\begin{tabular}{|c|c|c|c|c|}
\hline \multirow{2}{*}{$\begin{array}{l}\text { Death } \\
\text { SOFA }\end{array}$} & \multicolumn{3}{|c|}{ SSEPS } & \multirow[b]{2}{*}{ Total } \\
\hline & $<0.05$ & $0.05-0.2$ & $>0.2$ & \\
\hline$<0.05$ & 3 & 4 & 2 & 9 \\
\hline $0.05-0.2$ & 7 & 16 & 2 & 25 \\
\hline$>0.2$ & 2 & 5 & 7 & 14 \\
\hline \multirow[t]{2}{*}{ Total } & 12 & 25 & 11 & 48 \\
\hline & & & & $\mathrm{NRI}+=-0.125$ \\
\hline \multicolumn{5}{|l|}{ Alive } \\
\hline$<0.05$ & 69 & 31 & 1 & 101 \\
\hline $0.05-0.2$ & 39 & 30 & 7 & 76 \\
\hline$>0.2$ & 2 & 5 & 4 & 11 \\
\hline \multirow[t]{2}{*}{ Total } & 110 & 66 & 12 & 188 \\
\hline & & & & $\mathrm{NRI}-=0.037$ \\
\hline
\end{tabular}

$\mathrm{NRI}$, net reclassification index; SOFA, Sequential Organ Failure Assessment; SSEPS, Simple Sepsis Early Prognostic Score. admission, mechanical ventilation and shock in patients with sepsis during the early phases in the ED.

Age has been traditionally considered a risk factor for sepsis mortality probably due to the associated high rate of comorbidities, immune weakening, poorer preadmission status and high colonisation with Gram-negative bacteria. $^{23} 24$ Patients aged 80 and over have higher in-hospital mortality than those between 65 and 79 years, and age is strongly associated with sepsis in-hospital mortality. ${ }^{25}$ In our study, age was also independently associated with 28-day mortality.

Cardiac dysfunction due to irreversible cardiac damage in sepsis is one of the main causes of poor prognosis in patients with sepsis. Heart rate and left ventricular ejection fraction increase during the early stages of sepsis, but cardiac output decreases due to increased vascular permeability, decreased preload and decreased peripheral circulatory capacity, leading to insufficient perfusion of important organs. ${ }^{26}$ Although heart rate was not independently associated with sepsis mortality in our study, we included it in our SSEPS model (accounting for a maximum of 3 points of the total score) due to the simplicity of its measurement and to the published results that associate it with outcomes.

Respiratory rate and consciousness state are two indicators of the qSOFA score proven to be associated with prognosis of patients with sepsis. In our study, respiratory rate and altered consciousness were significantly higher in patients who died than in those who survived, and both variables were independently associated with risk of mortality in patients with sepsis. During sepsis, acute lung injury or acute respiratory distress syndrome occurs often due to diffuse alveolar damage caused by epithelial barrier and endothelial dysfunctions and by the resultant pulmonary oedema. ${ }^{27}$ In our study, the proportion of pulmonary infections was higher than those of other systems. Central nervous system dysfunction typically manifests as obtundation or delirium. ${ }^{28}$ We chose what we considered the most important and easy physiological indicators for our SSEPS and achieved good predictive ability with the system. 

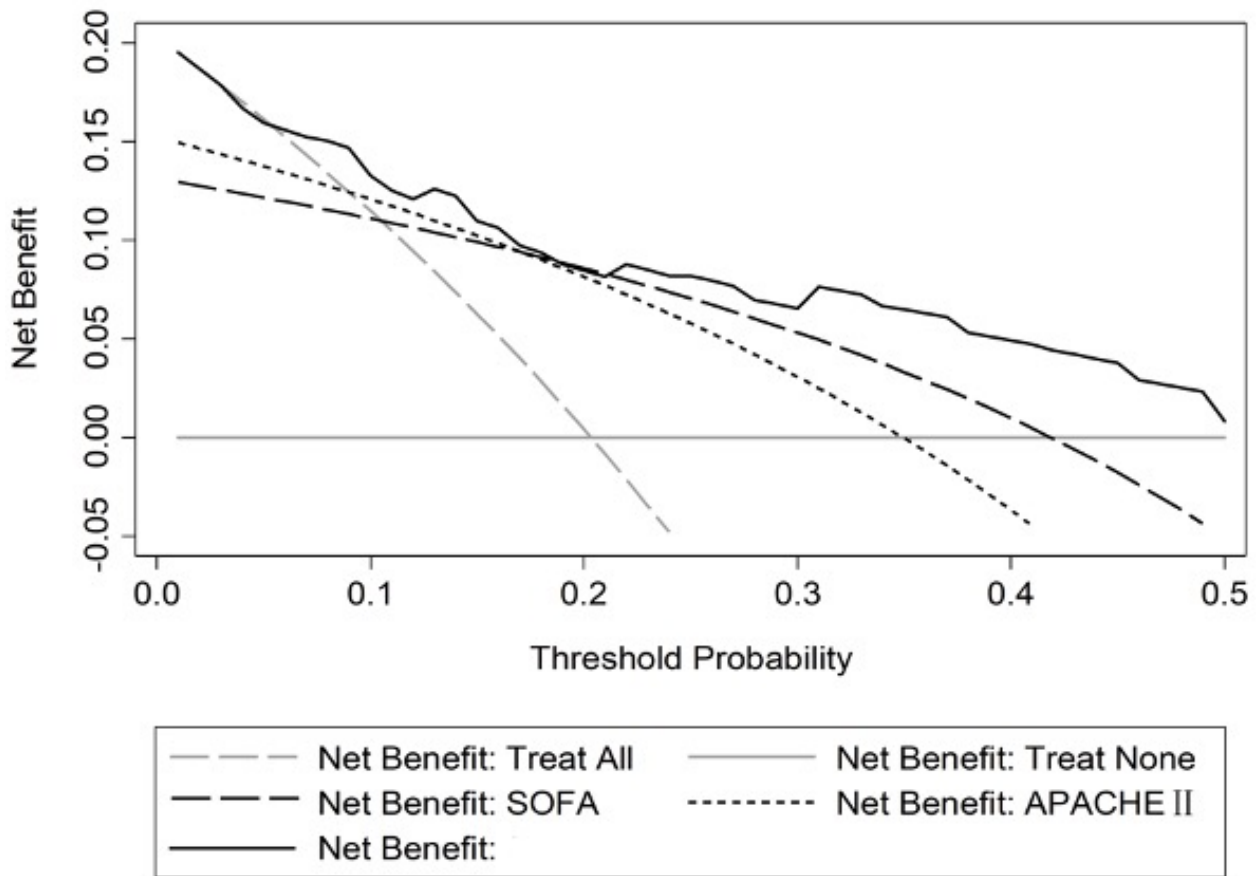

Figure 3 Decision curve analysis for SSEPS, SOFA and APACHE II in the validation group. APACHE II, Acute Physiology and Chronic Health Evaluation; SOFA, Sequential Organ Failure Assessment; SSEPS, Simple Sepsis Early Prognostic Score.

Our study also has limitations. First, it was a singlecentre, retrospective cohort study with a small population and we have not investigated the SSEPS for long-term outcomes. Second, we only recorded the variables for SSEPS on admission to our ED and we did not obtain recordings at different time points. A series evaluation of the SSEPS at different time points in our ED may provide more prognostic information. Third, the study lacked an external validation data set (only using internal validation), which may limit its rigour and confidence in findings.

\section{CONCLUSIONS}

SSEPS, a novel and easy-to-assess score, was an independent prognostic marker for patients with sepsis during the early phases in the ED, irrespective of severity of sepsis. The prognostic ability of the SSEPS in patients with sepsis was superior or not inferior to those of traditional prognostic sepsis scores. Therefore, SSEPS may be useful for emergency clinicians in risk stratification of patients with sepsis during the early phases of ED admission.

Acknowledgements The authors would like to acknowledge the participants and their families for taking part in the study.

Contributors All authors have contributed significantly to the manuscript. BL, DL and YCa designed the study. YCs, YJ, JY and QZ provided critical feedback and helped shape the research. JY, DL and YL analysed and interpreted the data. BL, DL and JY wrote the original draft of the manuscript. YCa wrote, reviewed and edited the manuscript. All authors read and approved the final version of the manuscript.

Funding This work was supported financially by grants from the Science Foundation of Science and Technology Department of Sichuan (no. 2018RZ0139 and 2019JDRC0105) and Science and Technology Benefit People Project of Chengdu Municipality(2016-HM02-00099-SF).
Competing interests None declared.

Patient consent for publication Not required.

Ethics approval This study complied with the Declaration of Helsinki and was approved by the Human Ethical Committee of the West China Hospital of Sichuan University (number: 2019 (373)).

Provenance and peer review Not commissioned; externally peer reviewed.

Data availability statement Data are available upon reasonable request. The data sets generated and/or analysed during the current study are not publicly available, in accordance with China Ethics Committee of Registering Clinical Trials, but are available from the coauthor on reasonable request.

Supplemental material This content has been supplied by the author(s). It has not been vetted by BMJ Publishing Group Limited (BMJ) and may not have been peer-reviewed. Any opinions or recommendations discussed are solely those of the author(s) and are not endorsed by BMJ. BMJ disclaims all liability and responsibility arising from any reliance placed on the content. Where the content includes any translated material, BMJ does not warrant the accuracy and reliability of the translations (including but not limited to local regulations, clinical guidelines, terminology, drug names and drug dosages), and is not responsible for any error and/or omissions arising from translation and adaptation or otherwise.

Open access This is an open access article distributed in accordance with the Creative Commons Attribution Non Commercial (CC BY-NC 4.0) license, which permits others to distribute, remix, adapt, build upon this work non-commercially, and license their derivative works on different terms, provided the original work is properly cited, appropriate credit is given, any changes made indicated, and the use is non-commercial. See: http://creativecommons.org/licenses/by-nc/4.0/.

ORCID iD

Bofu Liu http://orcid.org/0000-0001-9210-8462

\section{REFERENCES}

1 Singer M, Deutschman CS, Seymour CW, et al. The third International consensus definitions for sepsis and septic shock (Sepsis-3). JAMA 2016;315:801-10.

2 Seymour CW, Liu VX, Iwashyna TJ. Assessment of clinical criteria for sepsis: for the third International consensus definitions for sepsis and septic shock (Sepsis-3). Jama 2016;315:762-74. 
3 Wiersinga WJ, Leopold SJ, Cranendonk DR, et al. Host innate immune responses to sepsis. Virulence 2014:5:36-44.

4 Vincent J-L, Marshall JC, Ñamendys-Silva SA, et al. Assessment of the worldwide burden of critical illness: the intensive care over nations (icon) audit. The Lancet Respiratory Medicine 2014;2:380-6.

5 Thompson K, Venkatesh B, Finfer S. Sepsis and septic shock: current approaches to management. Intern Med J 2019;49:160-70.

6 Angus DC, Linde-Zwirble WT, Lidicker J, et al. Epidemiology of severe sepsis in the United States: analysis of incidence, outcome, and associated costs of care. Crit Care Med 2001;29:1303-10.

7 Rhodes A, Evans LE, Alhazzani W, et al. Surviving sepsis campaign: international guidelines for management of sepsis and septic shock: 2016. Intensive Care Med 2017;43:304-77.

8 Vincent JL, Moreno R, Takala J. The SOFA (sepsis-related organ failure assessment) score to describe organ dysfunction/failure. on behalf of the Working group on sepsis-related problems of the European Society of intensive care medicine. Intensive Care Med 1996;22:707-10.

9 Knaus WA, Draper EA, Wagner DP. Apache II: a severity of disease classification system. Crit Care Med 1985;13:818-29.

10 Roney JK, Whitley BE, Maples JC, et al. Modified early warning scoring (MEWS): evaluating the evidence for tool inclusion of sepsis screening criteria and impact on mortality and failure to rescue. $J$ Clin Nurs 2015;24:3343-54.

11 Shapiro NI, Wolfe RE, Moore RB, et al. Mortality in emergency department sepsis (MEDS) score: a prospectively derived and validated clinical prediction rule*. Crit Care Med 2003;31:670-5.

12 Fathi M, Markazi-Moghaddam N, Ramezankhani A. A systematic review on risk factors associated with sepsis in patients admitted to intensive care units. Australian Critical Care 2019;32:155-64.

13 Levey AS, Coresh J, Greene T, et al. Using standardized serum creatinine values in the modification of diet in renal disease study equation for estimating glomerular filtration rate. Ann Intern Med 2006;145:247-54.

14 Reith FCM, Van den Brande R, Synnot A, et al. The reliability of the Glasgow coma scale: a systematic review. Intensive Care Med 2016:42:3-15.

15 McKee AC, Daneshvar DH. The neuropathology of traumatic brain injury. Handb Clin Neurol 2015;127:45-66.
16 Demler OV, Pencina MJ, D'Agostino RB. Misuse of DeLong test to compare AUCs for nested models. Stat Med 2012;31:2577-87.

17 Pencina MJ, D'Agostino RB, Steyerberg EW. Extensions of net reclassification improvement calculations to measure usefulness of new biomarkers. Stat Med 2011;30:11-21.

18 Vickers AJ, Elkin EB. Decision curve analysis: a novel method for evaluating prediction models. Med Decis Making 2006;26:565-74.

19 Shashikumar SP, Stanley MD, Sadiq I, et al. Early sepsis detection in critical care patients using multiscale blood pressure and heart rate dynamics. J Electrocardiol 2017;50:739-43.

20 Cortés-Puch I, Hartog CS. Opening the debate on the new sepsis definition change is not necessarily progress: revision of the sepsis definition should be based on new scientific insights. Am J Respir Crit Care Med 2016;194:16-18.

21 Hilderink MJM, Roest AA, Hermans M, et al. Predictive accuracy and feasibility of risk stratification scores for 28-day mortality of patients with sepsis in an emergency department. European Journal of Emergency Medicine 2015;22:331-7.

22 Li D, Zhou Y, Yu J, et al. Evaluation of a novel prognostic score based on thrombosis and inflammation in patients with sepsis: a retrospective cohort study. Clin Chem Lab Med 2018;56:1182-92.

23 Brakenridge SC, Efron PA, Stortz JA, et al. The impact of age on the innate immune response and outcomes after severe sepsis/septic shock in trauma and surgical intensive care unit patients. J Trauma Acute Care Surg 2018;85:247-55.

24 Starr ME, Saito H. Sepsis in old age: review of human and animal studies. Aging Dis 2014;5:126-36.

25 Martin-Loeches I, Guia MC, Vallecoccia MS, et al. Risk factors for mortality in elderly and very elderly critically ill patients with sepsis: a prospective, observational, multicenter cohort study. Ann Intensive Care 2019;9:26.

26 Wilhelm J, Hettwer S, Schuermann M, et al. Severity of cardiac impairment in the early stage of community-acquired sepsis determines worse prognosis. Clin Res Cardiol 2013;102:735-44.

27 Butt Y, Kurdowska A, Allen TC. Acute lung injury: a clinical and molecular review. Arch Pathol Lab Med 2016;140:345-50.

28 Angus DC, van der Poll T. Severe sepsis and septic shock. $N$ Engl $J$ Med 2013;369:840-51. 